\title{
Transgressive phenotypes and generalist pollination in the floral evolution of Nicotiana polyploids
}

\author{
Elizabeth W. McCarthy 1,2,37, Mark W. Chase ${ }^{2}$, Sandra Knapp ${ }^{3}$, Amy Litt ${ }^{4}$, Andrew R. Leitch ${ }^{1}$ \\ and Steven C. Le Comber ${ }^{1 \star}$
}

\begin{abstract}
Polyploidy is an important driving force in angiosperm evolution, and much research has focused on genetic, epigenetic and transcriptomic responses to allopolyploidy. Nicotiana is an excellent system in which to study allopolyploidy because half of the species are allotetraploids of different ages, allowing us to examine the trajectory of floral evolution over time. Here, we study the effects of allopolyploidy on floral morphology in Nicotiana, using corolla tube measurements and geometric morphometrics to quantify petal shape. We show that polyploid morphological divergence from the intermediate phenotype expected (based on progenitor morphology) increases with time for floral limb shape and tube length, and that most polyploids are distinct or transgressive in at least one trait. In addition, we show that polyploids tend to evolve shorter and wider corolla tubes, suggesting that allopolyploidy could provide an escape from specialist pollination via reversion to more generalist pollination strategies.
\end{abstract}

P olyploidy, or whole genome duplication (WGD), is an important driving force in the evolution of angiosperms. Ancient polyploid events are shared by all seed plants, all angiosperms and all core eudicots, and there is evidence of multiple independent ancient WGDs across the angiosperms ${ }^{1-5}$. Polyploidy is associated with many genomic changes, including chromosomal rearrangements, retrotransposon activity, gene silencing and epigenetic changes, and it can also yield novel phenotypes, which may facilitate establishment of polyploids as species distinct from their progenitors ${ }^{1}$. Potentially, such phenotypic changes in floral structures may affect pollination. Flowers attract pollinators with colour, scent, shape, size of floral display, nectar and pollen rewards, and aspects such as corolla tube or nectar spur dimensions can influence pollinator access to any reward offered. Pollinator-mediated selection on floral traits has been shown in many studies ${ }^{6-10}$ and can even occur in generalist pollination systems because of different selective pressures from varying pollinator assemblages ${ }^{11}$.

Despite the importance of the changes following allopolyploidy, clear patterns of phenotypic evolution associated with allopolyploidy are hard to discern. In some cases, floral morphology in hybrids is intermediate between that of the parents, whereas in others phenotypes are transgressive (outside the range of the two parental species). For example, $\mathrm{F}_{4}$ hybrids between Nicotiana alata and $N$. forgetiana are intermediate in 12 corolla tube and floral limb (the portion of the flower that opens at anthesis) measurements ${ }^{12}$. However, other homoploid (diploid) hybrids display transgressive phenotypes, especially when alleles segregate in $F_{2}$ and subsequent generations ${ }^{13} . F_{1}$ hybrids between two species of Petunia with different corolla tube length and floral limb size have transgressively larger floral limbs, but intermediate corolla tube length; in the segregating $\mathrm{F}_{2}$ population, floral limb size is transgressive in both directions, and tube length can be transgressively larger ${ }^{14}$. In addition, autopolyploidy alone can yield floral changes even without the diversity generated by hybridization. For example, autotetraploids of Heuchera grossulariifolia have larger, differently shaped flowers than diploids and attract different floral visitors; 6 of 15 common floral visitor species preferred either diploid or autotetraploid flowers ${ }^{15}$.

Here, we study the effects of allopolyploidy on floral morphology in the genus Nicotiana. Nicotiana is an excellent system in which to study allopolyploidy because about half of its species are allotetraploids of different ages, including polyploids formed approximately $0.2,1,4.5$ and $10 \mathrm{Myr}$ ago; recent (synthetic) polyploids are also available (Table 1$)^{16-17}$. This series of ever older polyploid lineages enables us to examine both the consequences of polyploidy at formation and how polyploids may have diverged over time from an expected intermediate floral morphology based on that of known diploid progenitors ${ }^{16,18-21}$. Examples of floral morphology of Nicotiana polyploids and their diploid progenitors are found in Fig. 1. Our hypotheses are (1) polyploid floral divergence, from its predicted form at polyploid origin, will increase with age; (2) polyploid divergence in floral morphology will increase with greater progenitor morphological differentiation because there is the potential for greater floral variation on which selection/drift can act; and (3) polyploid species will diverge independently along lineage-specific trajectories.

\section{Results}

Floral morphological variation in Nicotiana. To examine the extent of variation present in floral limb shape, a geometric morphometric analysis of floral limb shape (Fig. 2a,b) was performed. The first two principal components of the resulting morphospace accounted for $58.18 \%$ and $20.33 \%$ of the variation present in the data, respectively. The morphospace of these two

${ }^{1}$ School of Biological and Chemical Sciences, Queen Mary University of London, Mile End Road, London E1 4NS, UK. ${ }^{2}$ Jodrell Laboratory, Royal Botanic Gardens, Kew, Richmond, TW9 3DS, UK. ${ }^{3}$ Natural History Museum, London SW7 5BD, UK. ${ }^{4}$ Department of Botany and Plant Sciences, University of California, Riverside, California 92521, USA. Present address: Department of Botany and Plant Sciences, University of California, Riverside, California 92521, USA. *e-mail: s.c.lecomber@qmul.ac.uk 
Table 1 | Polyploid and homoploid hybrid origins.

\begin{tabular}{|c|c|c|c|c|}
\hline Hybrid & Section & $\begin{array}{l}\text { Maternal } \\
\text { progenitor }\end{array}$ & Paternal progenitor & Age (millions of years) \\
\hline Synthetic N. tabacum QM & Synthetic Nicotiana & N. sylvestris & N. tomentosiformis & O (cross by K. Y. Lim, QMUL, UK) \\
\hline Synthetic N. tabacum TH37 & Synthetic Nicotiana & N. sylvestris & N. tomentosiformis & 0 (ref. 56) \\
\hline N. rustica & Rusticae & N. paniculata & N. undulata & $<0.2$ (refs 17,58$)$ \\
\hline Synthetic U×P & $\begin{array}{l}\text { Synthetic Paniculatae- } \\
\text { Undulatae }\end{array}$ & N. undulata & N. paniculata & O (diploid cross, A. Kovařík) \\
\hline $\begin{array}{l}\text { Synthetic N. rustica PUE1- } \\
\text { R10 } S_{0}\end{array}$ & Synthetic Rusticae & N. paniculata & N. undulata & O (synthetic PUE1 $F_{1}$ doubled, C. Mhiri) \\
\hline $\begin{array}{l}\text { Synthetic N. rustica PUE1-R1 } \\
\mathrm{S}_{1}\end{array}$ & Synthetic Rusticae & N. paniculata & N. undulata & O (putative $\mathrm{S}_{1}$ from doubled PUE1 $\mathrm{F}_{1}$ ) \\
\hline N. arentsii & Undulatae & N. undulata & N. wigandioides & $<0.2$ (refs 17,58$)$ \\
\hline N. nesophila & Repandae & N. sylvestris & N. obtusifolia & $\sim 4.5$ (ref. 16) \\
\hline N. stocktonii & Repandae & N. sylvestris & N. obtusifolia & $\sim 4.5$ (ref. 16) \\
\hline N. nudicaulis & Repandae & N. sylvestris & N. obtusifolia & $\sim 4.5$ (ref. 16) \\
\hline N. suaveolens & Suaveolentes & \multicolumn{2}{|c|}{$\begin{array}{l}\text { Progenitors: sections Noctiflorae, } \\
\text { Petunioides and Sylvestres }\end{array}$} & $\sim 10$ (refs $17,21,58$ ) \\
\hline N. glauca* & Noctiflorae-Petunioides & \multicolumn{2}{|c|}{$\begin{array}{l}\text { Progenitors: sections Noctiflorae and } \\
\text { Petunioides }\end{array}$} & N/A \\
\hline N. linearis* & Noctiflorae-Petunioides & \multicolumn{2}{|c|}{$\begin{array}{l}\text { Progenitors: sections Noctiflorae and } \\
\text { Petunioides }\end{array}$} & $\mathrm{N} / \mathrm{A}$ \\
\hline N. glutinosa* & Tomentosae-Undulatae & \multicolumn{2}{|c|}{$\begin{array}{l}\text { Progenitors: sections Tomentosae and } \\
\text { Undulatae }\end{array}$} & $\mathrm{N} / \mathrm{A}$ \\
\hline
\end{tabular}

*Homoploid hybrid evolution is difficult to detect and the age of origin of these hybrids have not been determined. QMUL, Queen Mary University of London.
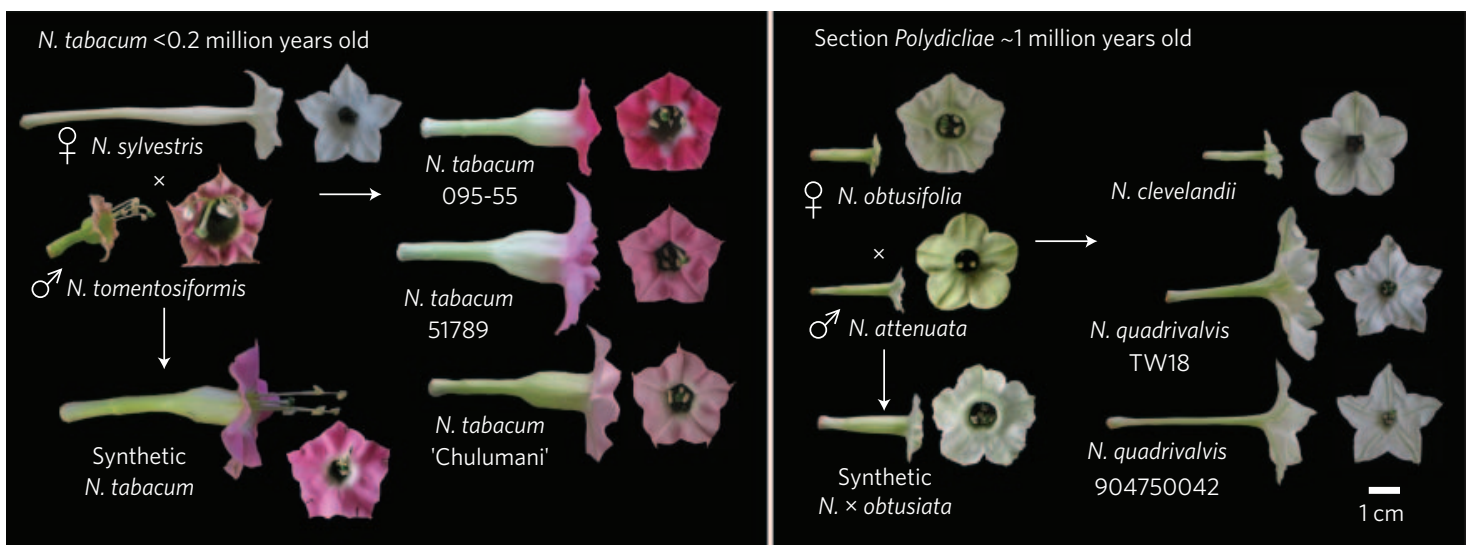

Figure 1 | Side and front floral morphology of $\boldsymbol{N}$. tabacum and section Polydicliae polyploids and their diploid progenitors. Side photographs to scale (scale bar, $1 \mathrm{~cm}$, lower right-hand corner), front photographs scaled to the same size. $\$$ and ${ }^{\star}$ symbols represent maternal and paternal progenitors, respectively.

principal components was defined by two characters on diagonal axes; on one axis, the floral outline changed from round to stellate, and, on the other, relative size of the corolla tube opening ranged from small to large. The top right-hand corner of the morphospace consisted of impossible shapes in which the internal landmarks that mark the tube opening cross, creating negative tube width (marked in grey in all figures). Figure $2 \mathrm{~b}$ shows the extent of the variation present in floral limb shape. Flowers from the same accession clustered in the first two principal components (Fig. 2b and Supplementary Fig. 1), but no clustering was seen in subsequent principal components (for example, PC3 and PC4, Supplementary Fig. 1).
Corolla tube length and width were measured to determine the 13 range of floral size. The accessions examined here clustered in the 14 scatter plot of tube width versus length (Fig. 2c). Tube length 15 ranged from 1.13 to $9.36 \mathrm{~cm}$, and tube width ranged from 0.1616 to $1.65 \mathrm{~cm}$.

Polyploid floral morphology. An intermediate phenotype between 18 those of the diploid progenitors is the simplest null hypothesis 19 for estimating polyploid phenotype immediately following 20 polyploidization. The extent of the evolution of polyploid floral 21 morphology was assessed by comparing each polyploid mean to 22 its progenitor midpoint (the mean of progenitor averages) and 23 

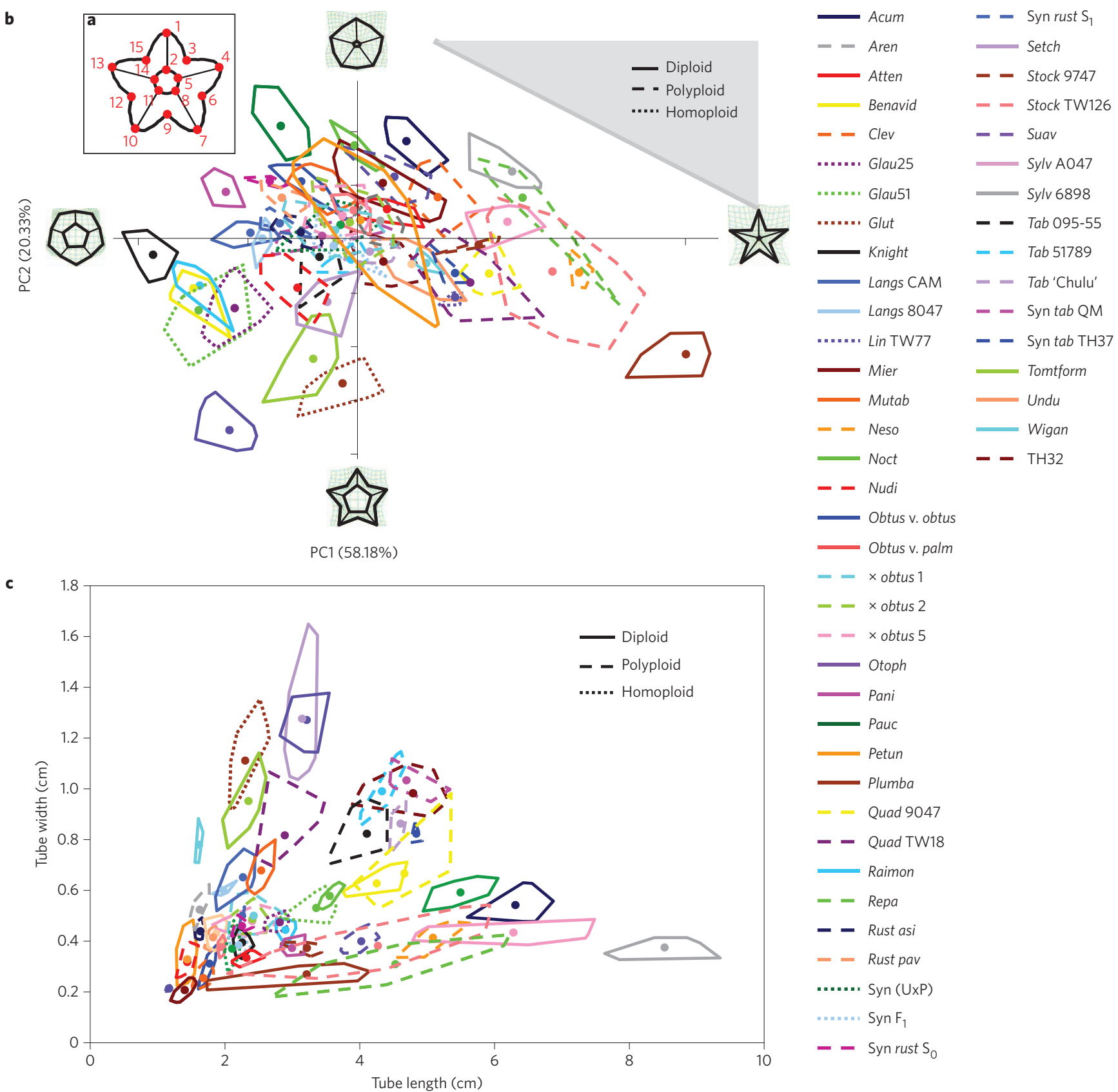

c

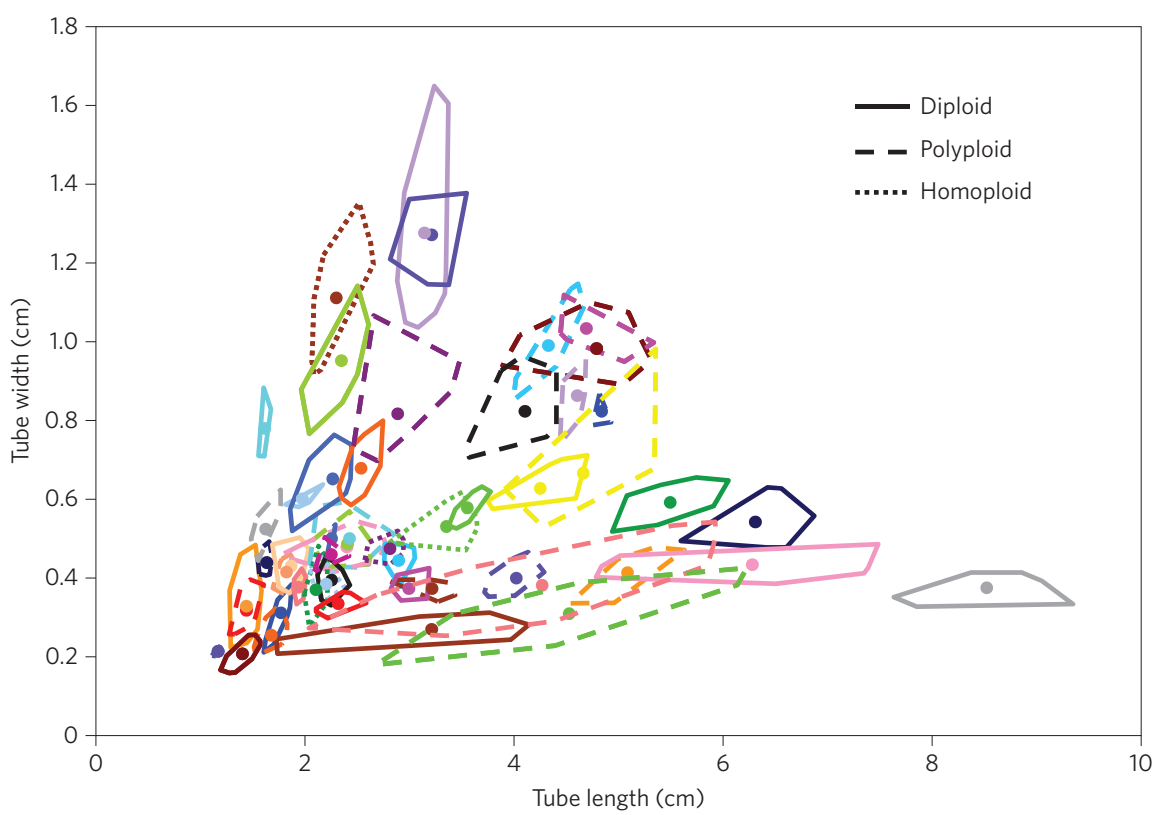

Figure 2 | Distribution of Nicotiana accessions in the floral limb shape morphospace and in tube length and width. a, Landmarks used to quantify floral limb shape via geometric morphometrics. b. First two components of the resulting morphospace from the geometric morphometric analysis, which consists of two morphological traits on diagonal axes: round to stellate floral outline on one and relatively small to large tube opening on the other. The images at the extremes of the axes show the relative warps of the landmarks at those points. The grey triangle in the right-hand corner represents impossible shapes. Convex polygons enclose the flower averages for each accession, representing the total variance of the accession; circles inside the polygons represent the mean. Diploid accessions have solid lines, polyploid accessions have dashed lines and homoploid or diploid hybrids have dotted lines. c, Corolla tube width is plotted against corolla tube length. Convex polygons and means are as described above. Accession abbreviations are found in the Methods.

1 progenitor midpoint range (estimated range of the progenitor midpoint based on the range of progenitors; see Methods for details). The oldest polyploid section, section Suaveolentes, was likely to have been formed before diversification of several Nicotiana sections ${ }^{21}$; therefore, a progenitor midpoint could not be calculated for $N$. suaveolens. Instead, this polyploid was compared with the morphology of all the species in its progenitor sections (Supplementary Fig. 2). Owing to the difference in comparison, this polyploid was left out of further calculations. In floral limb shape, $50 \%$ of polyploids overlapped with their progenitor midpoint range, $27 \%$ were distinct from the midpoint 11 range but within the combined ranges of their progenitors and 12 $23 \%$ had a transgressive phenotype outside the combined ranges 13 of their progenitors (Table 2, Fig. 3 and Supplementary Fig. 2; see 14 Methods for further explanation of 'overlapping', 'distinct' and 15 'transgressive'). Evidence from N. alata, N. forgetiana and their 16 hybrids showed that control of tube length development is not 17 genetically linked to that of tube width ${ }^{12,22}$. Therefore, we analysed 18 these characters independently. In tube length, 77\% of polyploids 19 overlapped with their progenitor midpoint range, $9 \%$ were distinct 20 
Table 2 | Polyploid divergence from the progenitor midpoint.

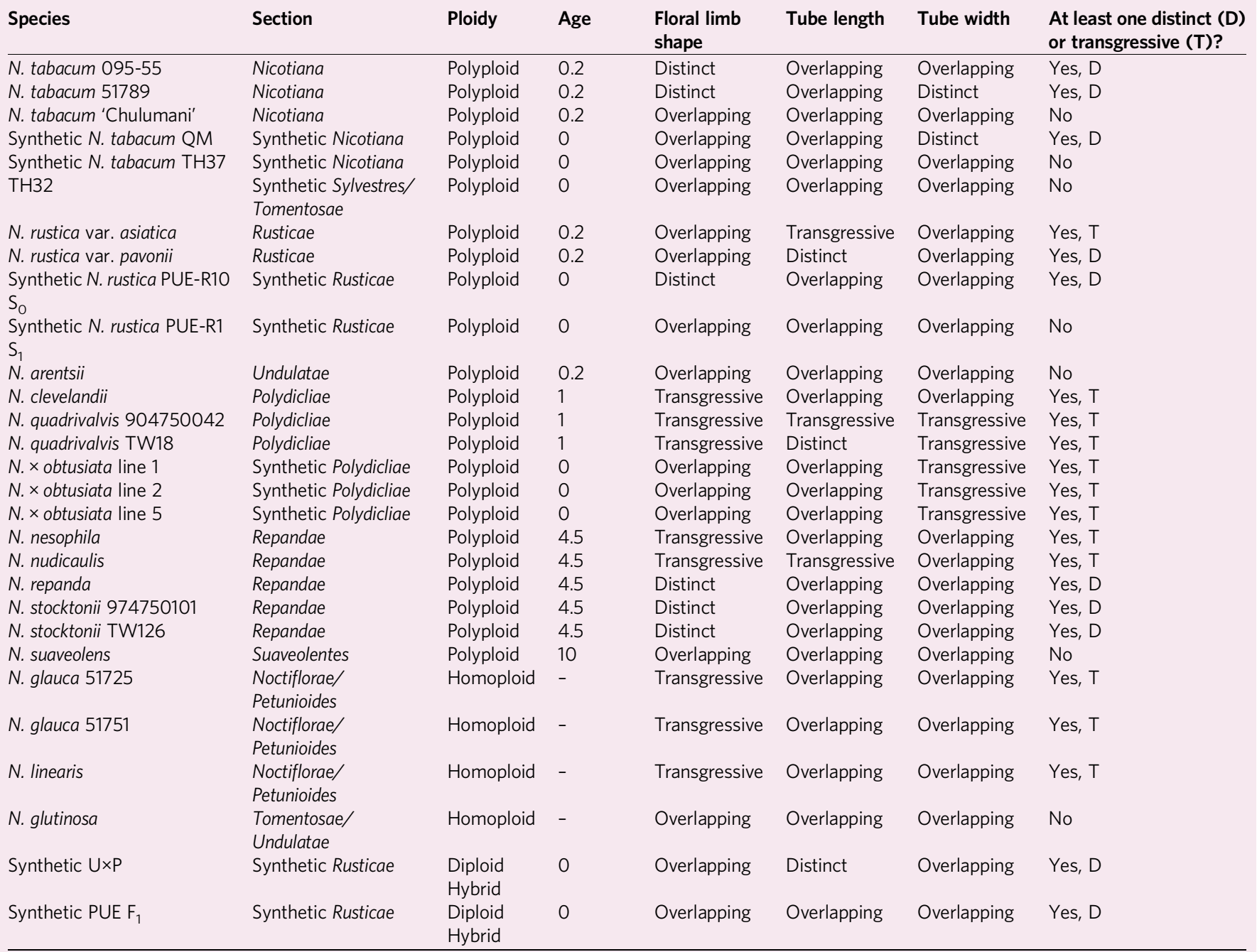

Overlapping, overlaps with progenitor midpoint range. Distinct, does not overlap with progenitor midpoint range. Transgressive, falls outside range of progenitors.

1 and 14\% were transgressive (Table 2, Fig. 3 and Supplementary 2 Fig. 2). In tube width, $68 \%$ of polyploids were overlapping, $9 \%$ are distinct and $23 \%$ are transgressive (Table 2, Fig. 3 and Supplementary Fig. 2). In examining all three traits together, most polyploids were either distinct or transgressive in at least one trait, whereas only $23 \%$ of polyploids overlapped with their progenitor midpoint range in all traits (Table 2).

Direction and degree of polyploid floral divergence from expectation. Directional distances of each polyploid mean from its progenitor midpoint were plotted with the origin as the progenitor midpoint (Fig. 4). Using this figure, the direction of change in morphology from predicted for each polyploid could be determined (based on the quadrant in which the polyploid falls). In floral limb shape, $70 \%$ of polyploids fell along the round to stellate floral axis, whereas $30 \%$ of polyploids diverged along the small to large tube opening axis (Fig. 4a). In tube length and width, $25 \%$ of polyploids had a longer and wider tube than expected, $50 \%$ had a shorter and wider tube and $25 \%$ had a shorter and narrower tube. No polyploid had a longer and narrower tube than expected (Fig. 4b). This distribution was significantly different from equal numbers of polyploids in each quadrant $\left(\chi^{2}=12\right.$, d.f. $\left.=3, P=0.0074\right)$, and polyploids tend to have shorter and wider tubes than expected.
Older polyploids tend to be more divergent from the progenitor 24 midpoint. To test whether younger polyploids had an intermediate 25 floral morphology between those of their diploid progenitors 26 whereas older polyploids had distinct or transgressive phenotypes, 27 we plotted the distance of each polyploid from its progenitor 28 midpoint against estimated polyploid age (Fig. 5).

In floral limb shape, distance from the midpoint increased 30 with polyploid age. An asymptotic curve was fitted to the data 31 $\left(y=0.0953451-5.97903 \times 10^{-16} / x\right.$; Fig. 5a). The shape of this 32 curve implies that divergence from the progenitor midpoint 33 occurs early in polyploid evolution. A Mood's median test on poly- 34 ploid distance from the midpoint revealed that the median distances 35 of the synthetic and young polyploids ( 0.2 million years old (myo)) 36 were significantly smaller than those of older polyploids (1 and 4.537 myo; $\chi^{2}=12.27$, d.f. $\left.=3, P<0.007\right)$. However, although young and 38 older polyploid groups were significantly different in distance, 39 their ranges overlapped such that the most divergent synthetic 40 and young polyploids were as divergent as the least divergent poly- 41 ploids of 1 and 4.5 myo (Fig. 5a).

In tube length, regression analysis showed that polyploid distance 43 from the midpoint and polyploid age were positively associated 44 $(F=4.63$; d.f. $=1, P=0.043)$; however, older polyploids showed 45 high variance in distance from the midpoint (Fig. 5b). This relation- 46 ship also confirms our hypothesis that older polyploids tend to be 47 

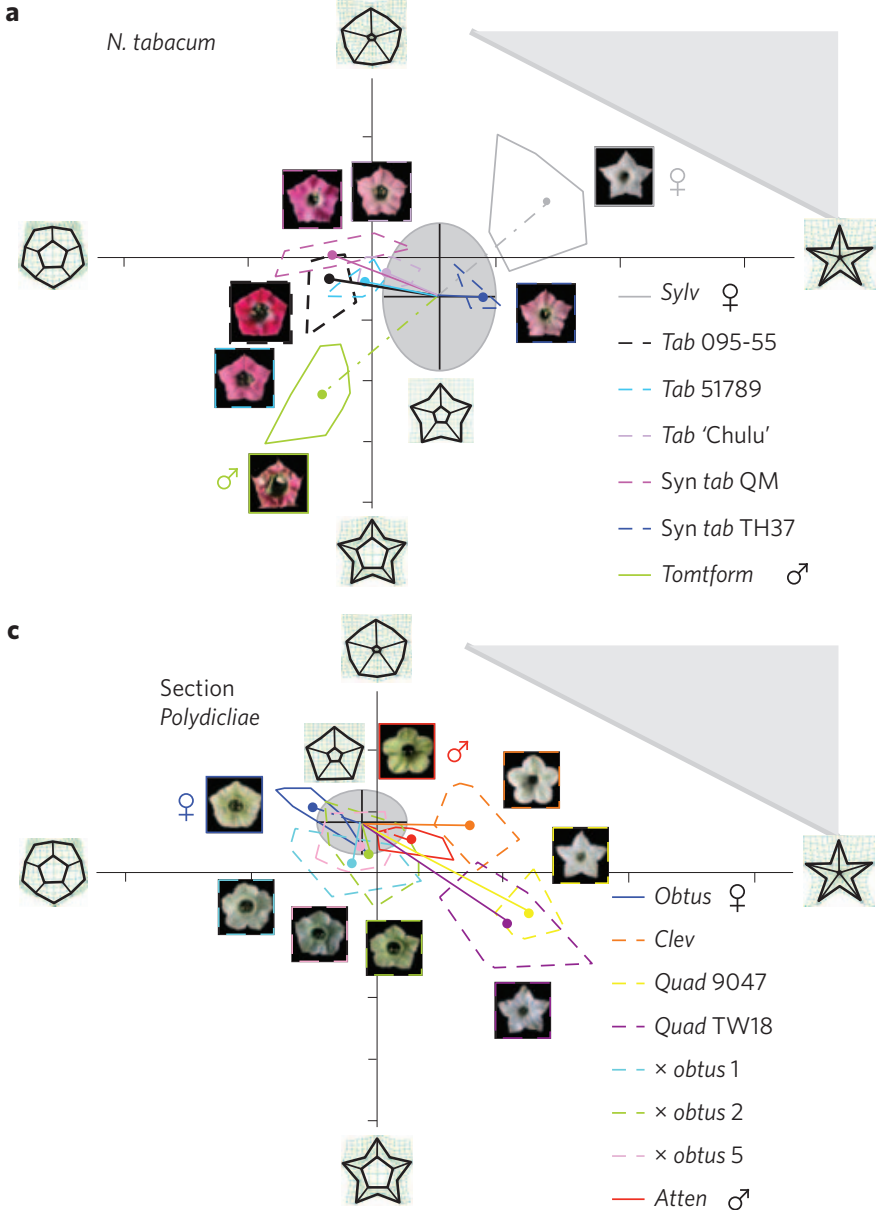

e

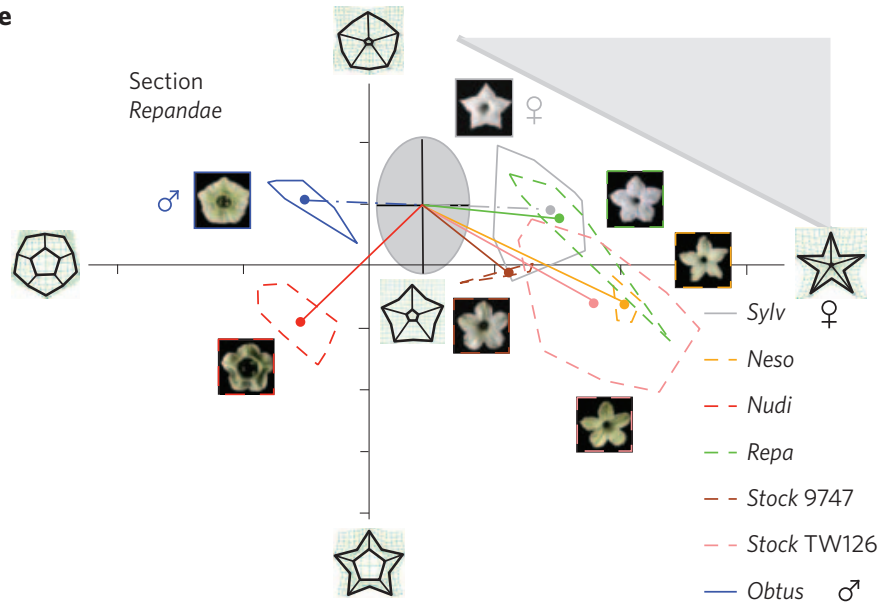

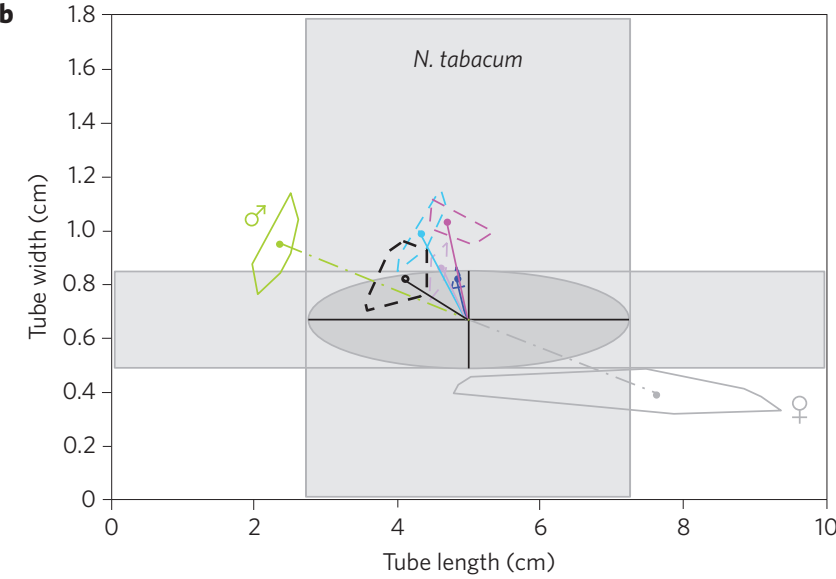

d

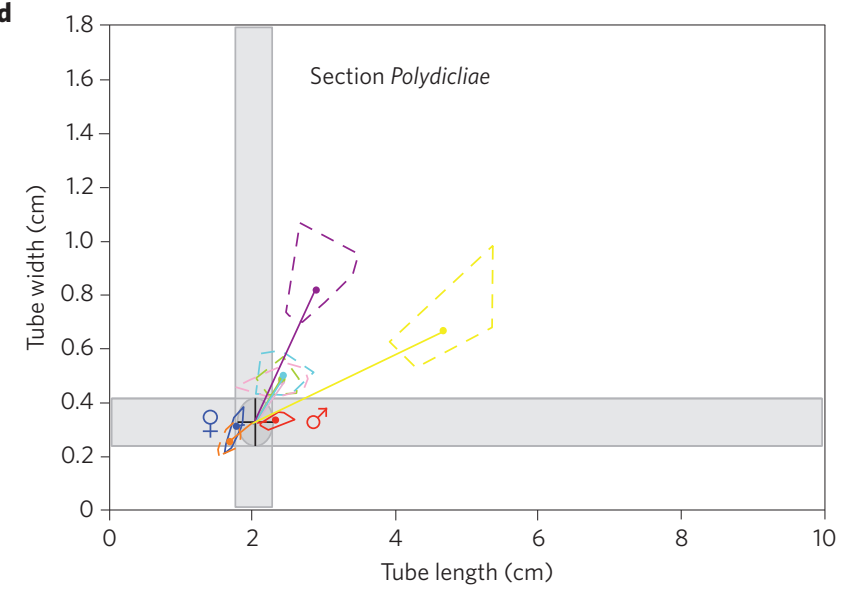

f

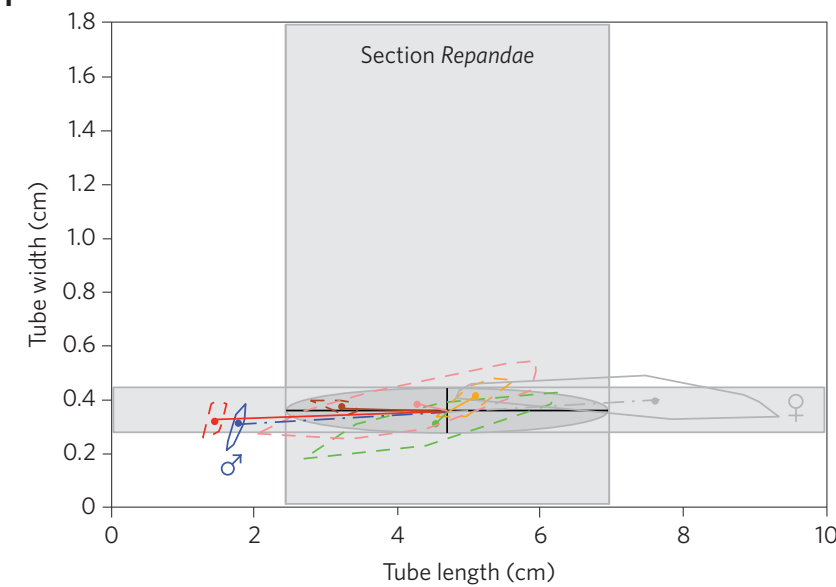

Figure 3 | Allotetraploid sections and their diploid progenitors in the floral limb morphospace and in tube length and width. a,b, N. tabacum. c,d, Section Polydicliae. e,f, Section Repandae. These figures compare polyploid morphology to that of their diploid progenitors and progenitor midpoint range (the expected polyploid morphology based on that of its progenitors). Convex polygons include the flower averages of each accession; diploids have solid lines and allotetraploids have dashed lines. The grey triangle in the top right-hand corner of the floral limb shape plots represents physically impossible shapes. The images at the extremes of the axes show the relative warps of the landmarks at those points. Grey ellipses and boxes represent the progenitor midpoint range. Flower photographs represent each accession and the coloured outline corresponds to the colour of the convex polygon. $\$$ and $\sigma^{\star}$ symbols denote maternal and paternal progenitors, respectively. Accession abbreviations are found in the Methods.

1 more divergent from the progenitor midpoint than younger ones. There was no significant relationship between distance from the progenitor midpoint in tube width and polyploid age $(F=2.57$, d.f. $=1, P=0.123$; Fig. 5c).

We also hypothesized that the morphological distance between diploid progenitor means might affect polyploid divergence.
However, there was no correlation between progenitor distance and polyploid distance from the midpoint in any of the traits examined (data not shown).

Homoploid hybrid floral morphology. Several species in Nicotiana 10 were shown to be homoploid hybrids ${ }^{19,23}$. However, the progenitors 11 

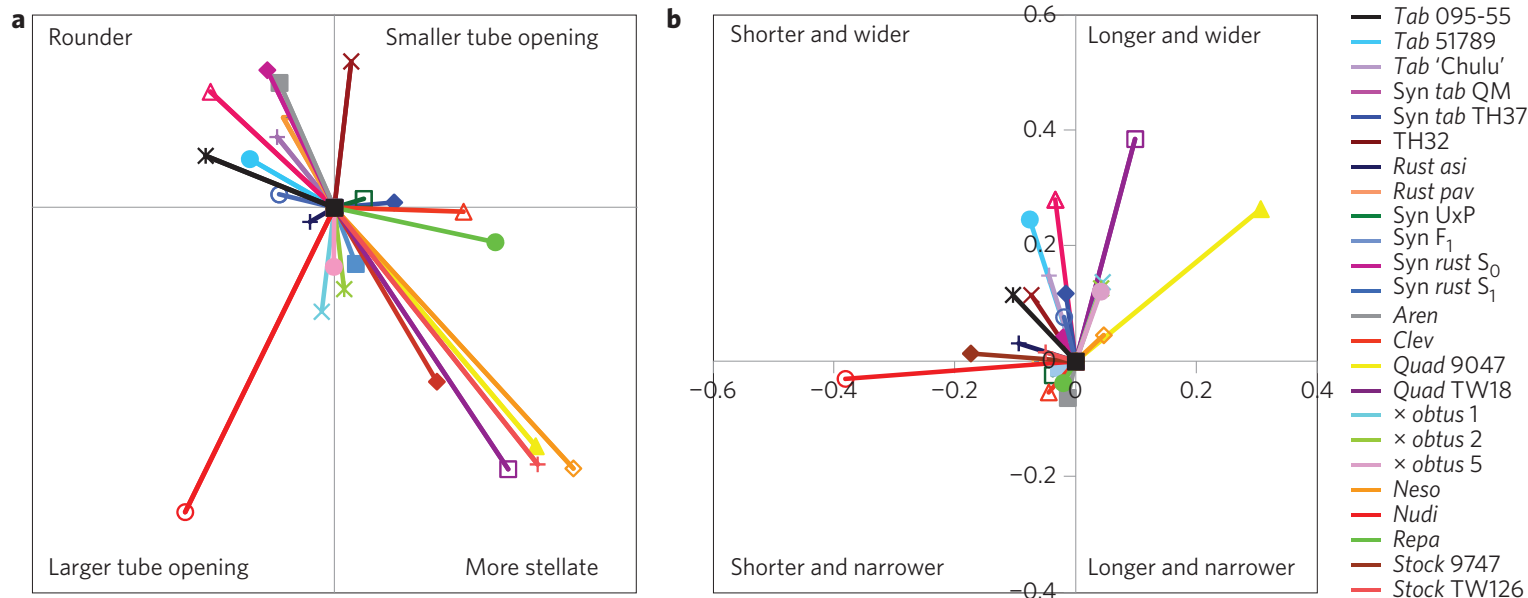

Figure 4 | Directional distance of polyploid means from the progenitor midpoint in floral limb shape and corolla tube length and width. These figures show trends in the floral evolution of polyploids by compiling the magnitude and direction of morphological change from the progenitor midpoint. The direction of morphological change is described by into which quadrant the polyploid falls. a, Smaller tube opening, rounder, larger tube opening or more stellate. $\mathbf{b}$, Longer and wider, shorter and wider, shorter and narrower, or longer and narrower. Accession abbreviations are found in the Methods.
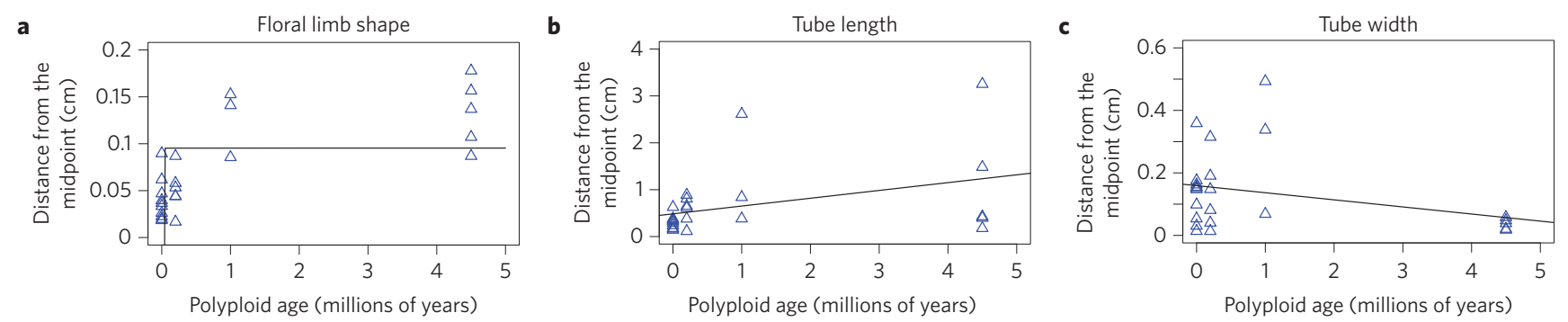

Figure 5 | Distance from the polyploid mean to the progenitor midpoint against estimated polyploid age for floral limb shape, corolla tube length and corolla tube width. a, Floral limb shape. An asymptotic curve was fitted to the data, which shows that distance from the progenitor midpoint in floral limb shape increases rapidly with polyploid age. $\mathbf{b}$, Corolla tube length. Regression analysis shows that polyploid distance from the progenitor midpoint in tube length is positively associated with polyploid age. c, Corolla tube width. Regression analysis shows no significant relationship between polyploid distance from the progenitor midpoint in tube width and polyploid age.

1 of these species were not represented by a single extant taxon; instead, it seemed that they were formed before the diversification of some of the sections of Nicotiana. Thus, we compared homoploid hybrid morphology with the total range of floral morphology found in the multiple species of their progenitor sections (Supplementary Fig. 3). In floral limb shape, half of homoploid hybrids were overlapping with their progenitor range and half were transgressive (Table 2 and Supplementary Figs 2 and 3). In tube length, $83 \%$ of homoploid hybrids were overlapping and $17 \%$ were distinct (Table 2 and Supplementary Figs 2 and 3). In tube width, all homoploid hybrids overlapped with their progenitor range (Table 2 and Supplementary Figs 2 and 3). Most homoploid hybrids were transgressive or distinct in at least one trait, whereas only $17 \%$ overlapped with their progenitor range in all traits examined.

\section{Discussion}

Our results show that divergence in floral morphology increases with polyploid age (Fig. 5) but not with increased morphological divergence between progenitor diploids (data not shown). In addition, we show that most polyploids are distinct or transgressive in at least one floral trait examined (Table 2) and that polyploids tend to have shorter and wider corolla tubes than expected (Fig. 4b).

3 Floral evolution following polyploidy. Divergence in floral limb shape proceeds rapidly following allopolyploidization and tends to increase over time (Fig. 5a). Divergence in tube length in 25 polyploids also tends to increase over time (Fig. 5b), but without 26 the initial rapid increase. Divergence in tube width, however, is 27 not associated with polyploid age (Fig. 5c). Differences in the 28 overall patterns of floral morphological divergence in polyploids 29 among characters suggest differing constraints on morphological 30 trait evolution. Floral limb shape seems to be the most 31 evolutionarily labile character as only $50 \%$ of polyploids overlap 32 with their progenitor midpoint range in floral limb shape 33 compared with $77 \%$ and $68 \%$ in tube length and width, 34 respectively (Table 2). Floral limb shape can also be highly 35 variable within some species (for example, $N$. glutinosa) ${ }^{24}, 36$ providing further evidence that the evolution of floral limb shape 37 is less constrained. Potentially the large variability in shape 38 among and within species arises because the floral limb is the 39 most prominent feature of the flower for attracting pollinators, 40 and it may be beneficial to be less constrained to facilitate rapid 41 response to changing selective pressures.

Tube length is an important factor in pollination in species with 43 nectar rewards, and it has been proposed that a fit between corolla 44 tube or nectar spur length and pollinator tongue length improves 45 both pollen removal and deposition ${ }^{25,26}$. Indeed, coevolution 46 between floral tube/spur length and tongue length, as proposed by 47 Darwin $^{27}$, has been reported in plant interactions for hawkmoths ${ }^{25}, 48$ long-tongued flies $^{28}$ and hummingbirds ${ }^{29}$. Within species (rep- 49 resented by a single population), floral traits that are involved in 50 
the fit between flower and pollinator are reported to have the smallest amount of phenotypic variation ${ }^{30}$. Pollinator-mediated selection on corolla tube length has been suggested from studies on six populations of Nicotiana glauca, and the strongest directional selection was proposed to occur in populations where there was the greatest mismatch between flowers and pollinators ${ }^{31}$. Taken together, this evidence suggests that tube length can be constrained by pollinator-mediated selection and is most likely to be under tight developmental control. Studies using $N$. alata, N. forgetiana and their hybrids show that corolla tube length, stamen length and carpel length are correlated ${ }^{12}$. In all natural polyploids examined here, stamens and carpels reach to the mouth of the corolla tube, even though some progenitors have exserted stamens. Synthetic $N$ tabacum QM is the exception (its paternal progenitor has exserted stamens), and the extent of stamen and carpel exsertion varies among synthetic lines (E.W. McCarthy et al., unpublished data). However, natural $N$. tabacum has inserted reproductive organs, perhaps suggesting that this trait is under selection in this species.

Tube width is also likely to be under pollinator-mediated selection. Relative to hummingbirds, bumblebees prefer wider corolla tubes $^{10,32}$. Narrower corolla tubes in hummingbird-pollinated Penstemon species are suggested to be due to both 'pro-bird' and 'anti-bee' adaptations ${ }^{10}$. In contrast, relative to hawkmoths, hummingbirds select for wider corolla tubes ${ }^{6,7}$. However, studies on flowers visited by short-billed and long-billed hummingbirds suggest that tube width is under greater selection from pollinators with shorter tongues and that changes in tube width may be a response to increases in corolla tube length ${ }^{33}$. Potentially, the evolution of a wider tube makes it possible for pollinators with shorter tongues to reach the nectar reward.

The divergence distance of the polyploids from the progenitor midpoint in tube width is not associated with polyploid age, largely because the oldest polyploids examined here have little divergence from expectation and the younger polyploids have a large range in divergence distances (Fig. 5c). The four allotetraploids of section Repandae, the oldest polyploids examined here, had a single origin ${ }^{16,19,20}$. One of these, $N$. nudicaulis, has a transgressively short corolla tube length, suggesting that even short-tongued pollinators can gain access to the nectar. The other three species, $N$. repanda, N. nesophila and N. stocktonii, are likely to be pollinated by nocturnal hawkmoths because they have long-tubed, white flowers that open at night. However, field pollination studies in Nicotiana are limited to a few diploid species, so the pollinators of these species have not been documented. Nicotiana nesophila and $N$. stocktonii are endemic to the isolated Revillagigedo Islands off the Pacific coast of Mexico $^{24}$. Hawkmoths are present on these islands ${ }^{34,35}$, but hummingbirds have not been recorded in recent surveys ${ }^{36}$. There was a single potential sighting of a hummingbird in a survey from $1897^{37}$. The lack of hummingbirds may have resulted in reduced selective pressure for wider tubes ${ }^{6,7}$.

Pollinators can also use shape cues as they forage. Bees can learn to distinguish between different convex shapes ${ }^{38}$ and prefer symmetry to asymmetry ${ }^{39}$. Hawkmoths prefer flowers with more dissected outlines (as opposed to convex) ${ }^{40}$. Shape, however, does not seem to be the most important cue in determining pollinator preference. Colour cues are more important than shape for bees ${ }^{41}$, and diurnal hawkmoths respond to both size and colour before shape while foraging ${ }^{42}$. Shape also appears to be less important than size for nocturnal hawkmoths because a preference for more deeply dissected floral outlines in Nicotiana section Alatae is no longer observed when floral shapes are standardized for surface area ${ }^{43}$. These observations that shape cues play a minor role in determining pollinator behaviour compared with other floral cues are consistent with our results that floral limb shape in polyploids is the most evolutionarily labile of the characters examined here (Table 2).
Polyploids tend to evolve features associated with generalist 67 pollination. Most polyploids have shorter and wider corolla tubes 68 than expected by their progenitor midpoints (Fig. 4b), indicating 69 a trend towards the evolution of features associated with more 70 generalist pollination (although the pattern of floral evolution in 71 polyploids differs somewhat if ancestral reconstructions of the 72 progenitor morphologies are considered, polyploids still diverge 73 towards shorter and wider tubes more often than is observed in 74 diploids; E. W. McCarthy et al., unpublished data). It was 75 previously found ${ }^{44}$ that the number of species of floral visitors 76 decreases as corolla tubes get longer but increases as tubes get 77 wider, suggesting that having shorter and wider corolla tubes can 78 in fact lead to more generalist pollination. Generalist pollination 79 may be advantageous to polyploids, especially during 80 establishment in a new or distinct niche from that of their 81 progenitors. Generalism may increase the probability that a 82 species will extend its range into new habitats and may decrease 83 the likelihood of extinction ${ }^{45-47}$. The adaptation of specialism to 84 certain pollinator types can hinder subsequent shifts to other 85 pollinators, suggesting that specialism can be an evolutionary 86 dead-end ${ }^{48-50}$. However, reversals in specialization do occur ${ }^{51}$, and 87 in some plant systems there is no evidence to link pollinator 88 specialization to decreased diversification ${ }^{50}$. Nevertheless, 89 polyploids tend to evolve shorter and wider tubes, suggesting that 90 allopolyploidy may provide an escape from specialization in 91 pollination (dead-end or otherwise), allowing lineages to diversify 92 by means of hybridization and WGD.

\section{Methods}

Plant growth. We grew plants in a greenhouse with a 16:8 h light/dark cycle that was 95 kept between 10 and $30^{\circ} \mathrm{C}$ year round with heaters, midterms and fans. The Nicotiana accessions examined here are found in Supplementary Table 1.

Flower photographs. We took photographs at anthesis with a Canon PowerShot A520. Nicotiana otophora is characterized by senescence of the floral limb before full 99 anthesis; therefore, it was photographed when the flower opened, but before the stamens dehisced. We took front and dissected view photographs for each flower (five replicates for each type per flower, five flowers per plant and five plants per accession), unless five plants were unavailable, in which case we took five to ten photographs for as many plants as possible (Supplementary Table 1).

We took front photographs by inserting the corolla tube into a hole in a piece of 105 cardboard so that the floral limb laid flat. In some species (N. setchellii,

N. langsdorffii and N. glauca), the floral limb forms a broad cup that cannot be flattened without disrupting the shape, so we placed flowers so the opening of the corolla tube lay against the cardboard and photographed directly above the corolla opening to minimize distortion of shape. For dissected photographs, we dissected flowers, removed the calyx and floral limb, and pinned the corolla tube open.

Geometric morphometric analysis. We used 15 landmarks to quantify floral limb 113 shape (Fig. 2a). We created a TPS file from front photographs using TPSUtil version 114 1.38 (ref. 52). We manually landmarked this series of photographs using TPSDig2 115 version 2.10 (ref. 53). We input these landmarked photographs into TPSRelw 116 version 1.45 (ref. 54), which calculated a consensus used as the reference specimen. 117 TPSRelw aligned all photographs to the reference, calculated partial warp scores for 118 each photograph and a principal components analysis was performed on partial 119 warp scores to calculate the relative warps of the dataset, which can be used to 120 visualize the shape variation present in the dataset.

We imported the principal component scores into Excel and calculated flower 122 and accession averages. We used scatterplots of both the first two principal 123 components and the third and fourth to examine the clustering of individual flowers 124 in the morphospace. We drew convex polygons around flower averages for each 125 accession in two-dimensional plots of the morphospace to delimit the portion of the 126 morphospace occupied by that accession. We marked the mean position of each 127 accession in the two-dimensional morphospace with a circle. We exported images 128 showing the relative warps of the landmarks from the extremes of the principal 129 components from TPSRelw to visualize the limits of the morphospace.

Metric measurements and analysis. We measured corolla tube length (from dissected photographs) and width (from landmarks 5 to 14) using Image J version 132 $1.42 \mathrm{q}$ (ref. 55). We calculated flower averages from five replicate photographs. We 133 made scatterplots of tube width versus length using flower averages and used convex 134 polygons and means to describe each accession. 
Calculation of progenitor midpoint values. We used the average of the progenitor means in the morphospace to calculate the progenitor midpoint. We calculated the range expected around the progenitor midpoint based on the ranges of the individual progenitors. We calculated the range of each progenitor for each trait and chose the larger range in each trait for a pair of progenitors to estimate the range around the progenitor midpoint. We delineated these chosen ranges in the morphospace as an ellipse with the progenitor midpoint at its centroid. The chosen range was divided in half and both added to and subtracted from the progenitor midpoint to calculate the ellipse. This 'progenitor midpoint range' is a conservative

10 measurement because it uses the maximum phenotypic variance of the progenitors.

1 Analyses of polyploid divergence. To analyse the extent of polyploid divergence from expected, we classified polyploids into three categories: overlapping (if a convex polygon enveloping all individuals of the polyploid accession overlapped with the progenitor midpoint range); distinct (if the polyploid polygon did not overlap with the progenitor midpoint range, but was within the combined ranges of the progenitors; or transgressive (if the polyploid polygon fell outside the combined ranges of the progenitors). We analysed tube length and width characters

independently because the development of tube length is not genetically linked to that of tube width ${ }^{12,22}$. Grey boxes delimit the progenitor midpoint range in tube width versus length figures. Owing to the nature of geometric morphometric analyses, principal component (PC) 1 and PC2 do not correspond to individual traits; therefore, we analysed floral limb shape using PC1 and PC2 together as two-dimensional coordinates. We calculated the distance of the polyploid mean from the progenitor midpoint for floral limb shape, tube length and tube width.

We plotted the distance between progenitor midpoint and polyploid mean in each floral character against both estimated polyploid age and distance between diploid progenitors in the same character. An asymptotic curve was fitted to the polyploid distance in floral limb shape versus polyploid age plot in Mathematica 5.0 (Wolfram Research Inc.). We performed regressions on all other plots using Minitab version 15.1 (Minitab, Inc.) and used a Mood's median test to determine significant differences between medians of each polyploid age group.

To examine overall trends in the evolution of floral morphology following polyploidy, we plotted the directional distance of the polyploid mean from the midpoint as a vector initiating at the origin, which represents the progenitor midpoint. The figure shows only the direction and magnitude of the change in polyploid morphology because progenitor midpoints from different pairs of progenitors, which are distinct in morphology, were translated to the origin. Because the range in tube length across the dataset is much larger that of tube width, we standardized the divergence measurements for tube length and width to proportional values (calculated by dividing each species mean by the largest species mean in each character). We used a goodness-of-fit chi-squared test to determine whether the distribution of polyploids into the four quadrants of the graph was significantly different from equal numbers in each.

Accession abbreviations. Accession abbreviations throughout the figures are as follows: acum, N. acuminata; aren, N. arentsii; atten, N. attenuata; benavid, N. benavidesii; clev, N. clevelandii; glau25, N. glauca 51725; glau51, N. glauca 51751; glut, N. glutinosa; knight, N. knightiana; langs CAM, N. langsdorffi CAM; langs 8047, N. langsdorffii 804750066; lin TW77, N. linearis TW77; mier, N. miersii; mutab, N. mutabilis; neso, N. nesophila; noct, N. noctiflora; nudi, N. nudicaulis; obtus v. obtus, N. obtusifolia var. obtusifolia TW143; obtus v. palm, N. obtusifolia var. palmeri; $\times$ obtus $1, N . \times$ obtusiata line $1 ; \times o b t u s 2, N . \times$ obtusiata line $2 ; \times$ obtus $5, N . \times$ obtusiata line 5; otoph, N. otophora; pani, N. paniculata; pauc, N. pauciflora; petun, N. petunioides; plumba, N. plumbaginifolia; quad 9047, N. quadrivalvis 904750042 quad TW18, N. quadrivalvis TW18; raimon, N. raimondii; repa, $N$. repanda; rust asi N. rustica var. asiatica; rust pav, N. rustica var. pavonii; syn $(\mathrm{U} \times \mathrm{P})$, synthetic $\mathrm{U} \times \mathrm{P}$; syn $\mathrm{F}_{1}$, synthetic PUE1 $\mathrm{F}_{1}$; syn rust $\mathrm{S}_{0}$, synthetic $N$. rustica PUE1-R10 $\mathrm{S}_{0}$; syn rust $\mathrm{S}_{1}$, synthetic N. rustica PUE1-R1 $\mathrm{S}_{1}$; setch, N. setchellii; stock 9747, N. stocktonii 974750101; stock TW126, N. stocktonii TW126; suav, N. suaveolens; sylv A047, N. sylvestris A04750326; sylv 6898, N. sylvestris 6898; tab 095-55, N. tabacum 095-55; tab 51789, N. tabacum 51789; tab 'Chulu', N. tabacum 'Chulumani'; syn tab QM, synthetic N. tabacum QM; syn tab TH37, synthetic N. tabacum TH37; tomtform, N. tomentosiformis; undu, N. undulata; wigan, N. wigandioides; TH32, TH32, synthetic $N$. sylvestris $\times N$. otophora polyploid.

\section{Received 8 April 2016; accepted 6 July 2016; published xx xx 2016}

\section{References}

1. Vanneste, K., Maere, S. \& Van de Peer, Y. Tangled up in two: a burst of genome duplications at the end of the Cretaceous and the consequences for plant evolution. Phil. Trans. R. Soc. B 369, 20130353 (2014)

2. Soltis, D. E., Visger, C. J. \& Soltis, P. S. The polyploidy revolution then.... and now: Stebbins revisited. Am. J. Bot. 101, 1057-1078 (2014).

3. Jiao, Y. et al. A genome triplication associated with early diversification of the core eudictos. Genome Biol. 13, R3 (2012).

4. Jiao, Y. et al. Ancestral polyploidy in seed plants and angiosperms. Nature 473, 97-100 (2011).
5. Renny-Byfield, S. \& Wendel, J. F. Doubling down on genomes: polyploidy and 76 crop plants. Am. J. Bot. 101, 1711-1725 (2014).

6. Campbell, D. R., Waser, N. M. \& Melendez-Ackerman, E. J. Analyzing pollinator-mediated selection in a plant hybrid zone: hummingbird visitation patterns on three spatial scales. Am. Nat. 149 (1997).

7. Melendez-Ackerman, E. \& Campbell, D. R. Adaptive significance of flower color and inter-trait correlations in an Ipomopsis hybrid zone. Evolution 52, 1293-1303 (1998)

8. Sletvold, N. \& Agren, J. Pollinator-mediated selection on floral display and spur length in the orchid Gymnadenia conopsea. Int. J. Plant Sci. 9, 999-1009 (2010). 85

9. Fenster, C. B. Selection on floral morphology by hummingbirds. Biotropica 23, 8 98-101 (1991)

10. Castellanos, M. C., Wilson, P. \& Thomson, J. D. 'Anti-bee' and 'pro-bird' changes during the evolution of hummingbird pollination in Penstemon flowers. 89 J. Evol. Biol. 17, 876-885 (2004).

11. Gomez, J. M. et al. Spatial variation in selection on corolla shape in a generalist 91 plant is promoted by the preference patterns of its local pollinators. Proc. R. Soc. B 92 275, 2241-2249 (2008).

12. Bissell, E. K. \& Diggle, P. K. Modular genetic architecture of floral morphology in 94 Nicotiana: quantitative genetic and comparative phenotypic approaches to floral 95 integration. J. Evol. Biol. 23, 1744-1758 (2010)

13. Rieseberg, L. H. \& Willis, J. H. Plant speciation. Science 317, 910-914 (2007).

14. Venail, J., Dell'Olivo, A. \& Kuhlemeier, C. Speciation genes in the genus Petunia. 9 Phil. Trans. R. Soc. B 365, 461-468 (2010).

15. Segraves, K. A. \& Thompson, J. N. Plant polyploidy and pollination: Floral traits 100 and insect visits to diploid and tetraploid Heuchera grossulariifolia. Evolution 53, 101 1114-1127 (1999).

16. Clarkson, J. J. et al. Long-term genome diploidization in allopolyploid Nicotiana 103 section Repandae (Solanaceae). New Phytol. 168, 241-252 (2005).

17. Leitch, I. J. et al. The ups and downs of genome size evolution in polyploid 105 species of Nicotiana (Solanaceae). Ann. Bot. 101, 805-814 (2008).

18. Chase, M. W. et al. Molecular systematics, GISH and the origin of hybrid taxa in 107 Nicotiana (Solanaceae). Ann. Bot. 92, 107-127 (2003).

19. Clarkson, J. J., Kelly, L. J., Leitch, A. R., Knapp, S. \& Chase, M. W. Nuclear 109 glutamine synthetase evolution in Nicotiana: phylogenetics and the origins of 110 allotetraploid and homoploid (diploid) hybrids. Mol. Phylogenet. Evol. 55, 111 99-112 (2010)

20. Clarkson, J. J. et al. Phylogenetic relationships in Nicotiana (Solanaceae) inferred 113 from multiple plastid DNA regions. Mol. Phylogenet. Evol. 33, 75-90 (2004). 114

21. Kelly, L. J., Leitch, A. R., Clarkson, J. J., Knapp, S. \& Chase, M. W. Reconstructing 115 the complex origin of wild allotetraploid tobaccos (Nicotiana section 116 Suaveolentes). Evolution 67, 80-94 (2013).

22. Bissell, E. K. \& Diggle, P. K. Floral morphology in Nicotiana: Architectural and temporal effects on phenotypic integration. Int. J. Plant Sci. 169, 225-240 (2008)

23. Kelly, L. J. et al. Intragenic recombination events and evidence for hybrid speciation in Nicotiana (Solanaceae). Mol. Biol. Evol. 27, 781-799 (2010).

24. Goodspeed, T. H. The Genus Nicotiana (Chronica Botanica, 1954).

25. Nilsson, L. A. The evolution of flowers with deep corolla tubes. Nature 334, 147-149 (1988)

26. Fulton, M. \& Hodges, S. A. Floral isolation between Aquilegia formosa and Aquilegia pubescens. Proc. R. Soc. Lond. B 266, 2247-2252 (1999). Darwin, C. On the Various Contrivances by which British and Foreign Orchids 128 are Fertilised by Insects (Murray, 1862).

28. Pauw, A., Stofberg, J. \& Waterman, R. J. Flies and flowers and Darwin's race. Evolution 63, 268-279 (2009)

29. Whittall, J. B. \& Hodges, S. A. Pollinator shifts drive increasingly long nectar spurs in columbine flowers. Nature 447, 706-709 (2007).

30. Cresswell, J. E Stabilizing selection and the structural variability of flowers within species. Ann. Bot. 81, 463-473 (1998).

31. Nattero, J., Sérsic, A. N. \& Cocucci, A. A. Patterns of contemporary phenotypic 136 selection and flower integration in the hummingbird-pollinated Nicotiana 137 glauca between populations with different flower-pollinator combinations. 138 Oikos 119, 852-863 (2010).

32. Galen, C. \& Cuba, J. Down the tube: Pollinators, predators, and the evolution of 140 flower shape in the alpine skypilot, Polemonium viscosum. Evolution 55, 1963-1971 (2001)

33. Temeles, E. J., Linhart, Y. B., Masonjones, M. \& Masonjones, H. D. The role of 143 flower width in hummingbird bill length-flower length relationships. Biotropica 144 34, 68-80 (2002).

34. Palacios-Vargas, J. G., Llampallas, J. \& Hogue, C. L. Preliminary list of the insects 146 and related terrestrial Athropoda of Socorro Island, Islas Revillagigedo, Mexico. 147 Bull. South. Calif. Acad. Sci. 81, 138-147 (1982).

35. Hanna, G. D. Expedition to the Revillagigedo Islands, Mexico, in 1925. Proc. Calif. Acad. Sci. XV, 1-113 (1926).

36. Hahn, I. J., Hogeback, S., Romer, U. \& Vergara, P. M. Biodiversity and biogeography of birds in Pacific Mexico along an isolation gradient from mainland Chamela via coastal Marias to oceanic Revillagigedo Islands. Vertebr. Zool. 62, 123-144 (2012). 147 (a) 138
139 (10)

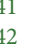
(n)

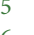


37. Anthony, A. W. Avifauna of the Revillagigedo Islands. Auk 15, 311-318 (1898).

38. De Ibarra, N. H. \& Giurfa, M. Discrimination of closed coloured shapes by honeybees requires only contrast to the long wavelength receptor type. Anim. Behav. 66, 903-910 (2003).

39. Giurfa, M., Eichmann, B. \& Menzel, R. Symmetry perception in an insect. Nature 382, 458-461 (1996).

40. Herrera, C. M. Selection on complexity of corolla outline in a hawkmothpollinated violet. Evol. Trends Plants 7, 9-13 (1993).

41. Lehrer, M. \& Campan, R. Generalization of convex shapes by bees: what are shapes made of? J. Exp. Biol. 208, 3233-3247 (2005).

42. Kelber, A. Innate preferences for flower features in the hawkmoth Macroglossum stellatarum. J. Exp. Biol. 200, 827-836 (1997).

43. Kaczorowski, R. L., Seliger, A. R., Gaskett, A. C., Wigsten, S. K. \& Raguso, R. A. Corolla shape vs. size in flower choice by a nocturnal hawkmoth pollinator. Funct. Ecol. 26, 577-587 (2012).

44. Stang, M., Klinkhamer, P. G. L. \& Van Der Meijden, E. Size constraints and flower abundance determine the number of interactions in a plant-flower visitor web. Oikos 112, 111-121 (2006).

45. Bond, W. J. Do mutualisms matter? Assessing the impact of pollinator and disperser disruption on plant extinction. Phil. Trans. R. Soc. Lond. B 344, 83-90 (1994).

46. Zayed, A. et al. Increased genetic differentiation in a specialist versus a generalist bee: implications for conservation. Conserv. Genet. 6, 1017-1026 (2005).

47. Johnson, S. D. \& Steiner, K. E. Generalization versus specialization in plant pollination systems. Trends Ecol. Evolut. 15, 140-143 (2000).

48. Wilson, P., Castellanos, M. C., Wolfe, A. D. \& Thomson, J. D. in Plant-pollinator Interactions: From Specialization to Generalization (eds Waser, N. M. \& Ollerton, J.) 47-68 (University of Chicago Press, 2006).

49. Tripp, E. A. \& Manos, P. S. Is floral specialization an evolutionary dead-end? Pollination system transitions in Ruellia (Acanthaceae). Evolution 62, 1712-1736 (2008).

50. Day, E. H., Hua, X. \& Bromham, L. Is specialization and evolutionary dead end? Testing for differences in speciation, extinction and trait transition rates across diverse phylogenies of specialists and generalists. J. Evolut. Biol. 29, 1257-1267 (2016).
51. Vamosi, J. C., Armbruster, W. S. \& Renner, S. S. Evolutionary ecology of specialization: insights from phylogenetic analysis. Proc. R. Soc. B 281, 20142004 (2014).

52. SUNY. tps Utility program. Ecology and Evolution (Stony Brook, NY, 2006).

53. SUNY. tpsDig. Ecology and Evolution (Stony Brook, NY, 2006).

54. SUNY. Relative warps. Ecology and Evolution (Stony Brook, NY, 2007).

55. U.S. National Institutes of Health. ImageJ (Bethesda, MD, 1997-2010).

56. Burk, L. G. Partial self-fertility in a theoretical amphiploid progenitor of N. tabacum. J Hered. 64, 348-350 (1973).

57. Moon, H. S., Nicholson, J. S. \& Lewis, R. S. Use of transferable Nicotiana Nicotiana. Genome 51, 547-559 (2008).

58. Clarkson, J. J. Nicotiana (Solanaceae): Insights from Molecular Phylogenetics and 48 Cytogenetics $\mathrm{PhD}$ thesis, Queen Mary Univ. London (2006).

59. Anssour, S. et al. Phenotypic, genetic and genomic consequences of natural and 50 synthetic polyploidization of Nicotiana attenuata and Nicotiana obtusifolia. Ann. Bot. 103, 1207-1217 (2009).

\section{Acknowledgements}

We thank K. Yoong Lim, A. Kovařík, C. Mhiri and I. T. Baldwin for providing us with synthetic polyploids and homoploids. This work was supported by the Natural Environment Research Council (NE/C511964/1 to A.R.L. and M.C.) and the Overseas Research Students Awards Scheme to E.W.M.

\section{Author contributions}

E.M., M.C., S.K., A.R.L. and S.L. designed the research. E.M. collected the data and 59 performed most of the analyses. S.L. performed analyses in Mathematica. E.M., A.R.L. and 60 S.L. wrote the manuscript with help from M.C., S.K. and A.L.

\section{Additional information}

Supplementary information is available online. Reprints and permissions information is 63 available online at www.nature.com/reprints. Correspondence and requests for materials should 64 be addressed to S.C.L.C.

\section{Competing interests}

The authors declare no competing financial interests. 50 . 61 64
65 
Journal: $\quad$ NPLANTS

Article ID: $\quad$ nplants.2016.119

Article Title: Transgressive phenotypes and generalist pollination in the floral evolution of Nicotiana polyploids Author(s): $\quad$ Elizabeth W. McCarthy et al.

\begin{tabular}{|l|l|l|}
\hline $\begin{array}{l}\text { Query } \\
\text { no. }\end{array}$ & Query & Response \\
\hline 1 & $\begin{array}{l}\text { The title has been changed to avoid the use of } \\
\text { punctuation. }\end{array}$ & \\
\hline 2 & $\begin{array}{l}\text { 'The first two principal components...' What are the two } \\
\text { components? }\end{array}$ & \\
\hline 3 & Please check the edits to Figures 3 to 5 captions. & \\
\hline
\end{tabular}

